\title{
Clinical significance of some new thyroid parameters
}

\author{
Florentina Rus-Hrincu', Alexandra Totan ${ }^{1}$, Anca-loana Belusica ${ }^{2}$, \\ Iulia-Ioana Stanescu', Maria Greabu' ${ }^{1}$ \\ ${ }^{1}$ Department of Biochemistry, "Carol Davila" University of Medicine and Pharmacy, \\ Bucharest, Romania \\ ${ }^{2} \mathrm{CMI}$ Anca Belusica, Bucharest, Romania
}

\begin{abstract}
There is increasing interest in the development of new and easily applied markers that can predict thyroid malfunction. Clinicians need low-cost, faster and minimal invasive methods of diagnosis. In this study we will analyze biochemical markers involved in thyroid carcinoma, thyroid disorders and the antioxidant role of TH. Studies showed a correlation between thyroid disorders and OS markers. Numerous studies suggested that all tree routine parameters, TSH, FT4 and FT3, must be viewed together to be relevant for diagnose thyroid affections. Some authors even proposed lowering the reference level of TSH to define subclinical hypothyroidism. Another studies suggested that TG level, thyroglobulin autoantibodies and higher level of TSH could be associated with thyroid malignancy. Also, the ratio between $\mathrm{TSH}$ and $\mathrm{Tg}$ should alert the clinician to the possibility of thyroid carcinoma. Another important markers in thyroid pathology could be DIOs, selenoenzimes with crucial role in $\mathrm{TH}$ synthesis.

Recent studies confirmed that thyrocyte is under OS, and in the last years begun to be clarified the source of thyroid hydrogen peroxide involved in hormone biosynthesis and cell damage. Hydrogen peroxide necessary for hormone biosynthesis it is produced in a reaction catalyzed by DUOX1 and DUOX2. Hydrogen peroxide generated by DUOX inhibit TPO activity due to oxidative damage to the enzyme. This could increase OS in thyrocyte. Another enzyme it is believed that has a pivotal role in hydrogen peroxide synthesis and that is SOD 3.

OS is related to hormonal derangement and systemic inflammation. Studies linked OS with hyperthyroidism and hypothyroidism, and even suggested that TH can produce DNA damage by acting as oxidants.

Important markers could help thyroid disorders biochemical diagnosis and patient management in a minimal invasive way. Beside routine parameters like TSH, FT4, FT3, could be useful for the clinician other parameters such as $\mathrm{Tg}$ level, ratio between

TSH and Tg, DIOs expression, TPO level and OS markers: NADPH oxidases, DUOX and SOD.
\end{abstract}

Keywords: thyroid, thyroid hormones, deiodinase, dual oxidase, thyroglobulin, oxidative stress

\section{INTRODUCTION}

The human body is a highly complex, biological system where normality and variation are tightly controlled by various mechanisms (1). The hypothalamus-pituitary-thyroid-axis is concerned with energy metabolism and multiple specific functions within the organism (1).

Currently, there is increasing interest in the development of new and easily applied markers that have the potential to enhance our ability to predict thyroid malignancy (2-4). Today, preoperative serum markers like thyroglobulin $(\mathrm{Tg})$, thyroid stim- ulating hormone (TSH) and thyroid antibodies are attracting growing interest among clinicians because they are fairly accessible tests, faster and low-cost, some of them even covered by health insurance, by contrast with other laborious or invasive methods (5).

Studies showed that thyroid cancer is the most frequent cancer among endocrine tumors, and its incidence has been greatly increasing in many countries $(6,7)$. In the last decade, an effort has been made to predict the risk of thyroid cancer using the markers of thyroid function/autoimmunity $(7-11)$. 
In this study we will analyze biochemical markers involved in thyroid cancer, thyroid disorders and the antioxidant role of thyroid hormones (TH). There is proof that thyroid disorders and oxidative stress (OS) markers such as superoxide dismutase (SOD), dual oxidase (DUOX) or NADPH oxidases are correlated.

\section{THYROID PARAMETERS}

The human thyroid gland produces and then releases into the circulation a large amount of thyroxine (T4) and a lesser proportion of triiodothyronine (T3). Only very small concentrations exist as the respective unbound free forms, free T3 (FT3) significantly more than free T4 (FT4). The two hormones are interrelated by conversion of $\mathrm{T} 4$ into $\mathrm{T} 3$ by enzymatic 5 'monodeiodination $(1,12)$.

Homeostatic interrelations, equilibria, biological variation and set points between TSH, FT4, and FT3 become relevant for the observed expression of multivariate normality and relational stability in thyroid health. FT3 becomes a primary target for system stability $(1,8)$. All three routine thyroid parameters (THS, FT4, FT3) must be viewed together in order to create an appropriate image of the possible thyroidal affection $(13,14)$. Some authors even proposed to lower the conventional reference level for TSH to $2 \mathrm{mUI} / 1$. This level it should define subclinical hypothyroidism $(15,16)$.

Another parameter important in diagnosis of thyroid affections is the Tg level. Studies from the last decade are focused to find new methods to identify preoperative malignancy in thyroid nodules by measuring the Tg level $(11,17,18)$. This method is easily accessible and low-cost comparable with classics methods of diagnostics.

Because association between thyroid cancer and thyroid autoimmune disease, some studies suggested that thyroid autoantibodies could be used as predictors of thyroid cancer risk (11).

Other studies showed that higher levels of TSH could be associated with thyroid malignancy $(9,19)$.

A group of scientists from Taiwan studied the incidence of cancer patients with hyperthyroidism and found that they have increased risk of thyroid cancer. They observed the duration of hyperthyroidism influenced the risk of thyroid cancer (10).

Cho et al. (7) reported that the levels of TSH and $\mathrm{Tg}$ were associated with thyroid cancer risk only among smokers.

In another study, Jing Qin et al. demonstrated that the prevalence of differentiated thyroid carcinoma was higher in patients with elevated anti-thy- roglobulin antibodies (TgAb) (over $40 \mathrm{IU} / \mathrm{ml}$ ). Levels of $\mathrm{TgAb} \geq 40 \mathrm{IU} / \mathrm{ml}$ correlated with elevated TSH levels might be a predictive marker for differentiated thyroid carcinoma, but they didn't observe a correlation between lymph node metastasis or advance cancer stages and elevated $\mathrm{TgAb}$ or anti-thyroid peroxidase antibodies (TPOAb) levels (20).

Several studies showed that the simultaneously increased levels of both TSH and Tg are not useful in predicting thyroid malignancy in the preoperative period (21-24).

Serum Tg level it is not specific for thyroid cancer but elevated levels can be detected in other proliferative thyroid disorders $(23,24)$.

In recent studies was analyzed the ratio between $\mathrm{TSH}$ and $\mathrm{Tg}(20,24)$ and showed that TSH:Tg ratio is significantly increased in patients with thyroid cancer, and should alert the clinician to the possibility of thyroid cancer $(25,26)$.

\section{IODOTHYRONINE DEIODINASES}

Iodothyronine deiodinases are a family of enzymes selenocysteine dependent that catalyze the stereospecific removal of iodine atoms from one aromatic ring from $\mathrm{T} 4$, generating the active and inactive isomers of $\mathrm{T} 3$ and 3,3'-diiodothyronine (T2) (27). Conversion of T4 to T3 requires removal of one iodine atom from the outer ring of T4. Deiodination of the outer ring is mediated by deiodinases type I (DIO1) and II (DIO2) (12). While deiodinases type III (DIO3) mediates deiodination in the inner ring and converts $\mathrm{T} 4$ to inactive metabolites 3,3',5'-triiodotyronine or reverse T3 (rT3) and T2 (28). DIO1 has lower affinities for the substrates than DIO2 and seems to be a scavenger enzyme, involved in iodine recycling. DIO2 is located in the endoplasmic reticulum and plays the primary role in the conversion of T4 to T3 (Fig. 1).

The balance between DIO2 and DIO3 activities seems to be an important factor in determining the amount of T3 available to bind the nuclear receptors $(27,29)$.

Different mechanisms regulate the expression of DIO genes (DIO1, DIO2 and DIO3). One of them is the levels of TH: hypothyroidism increase the activity of DIO2 while hyperthyroidism suppresses DIO2 activity and expression (27). Another mechanism of regulation of DIO activity is the ubiquitination of the enzymes, which can be reversible to assure the appropriate protein homeostasis (30).

DIO1 is primarily expressed in the liver and kidney and has a much lower affinity for T4 than DIO2 


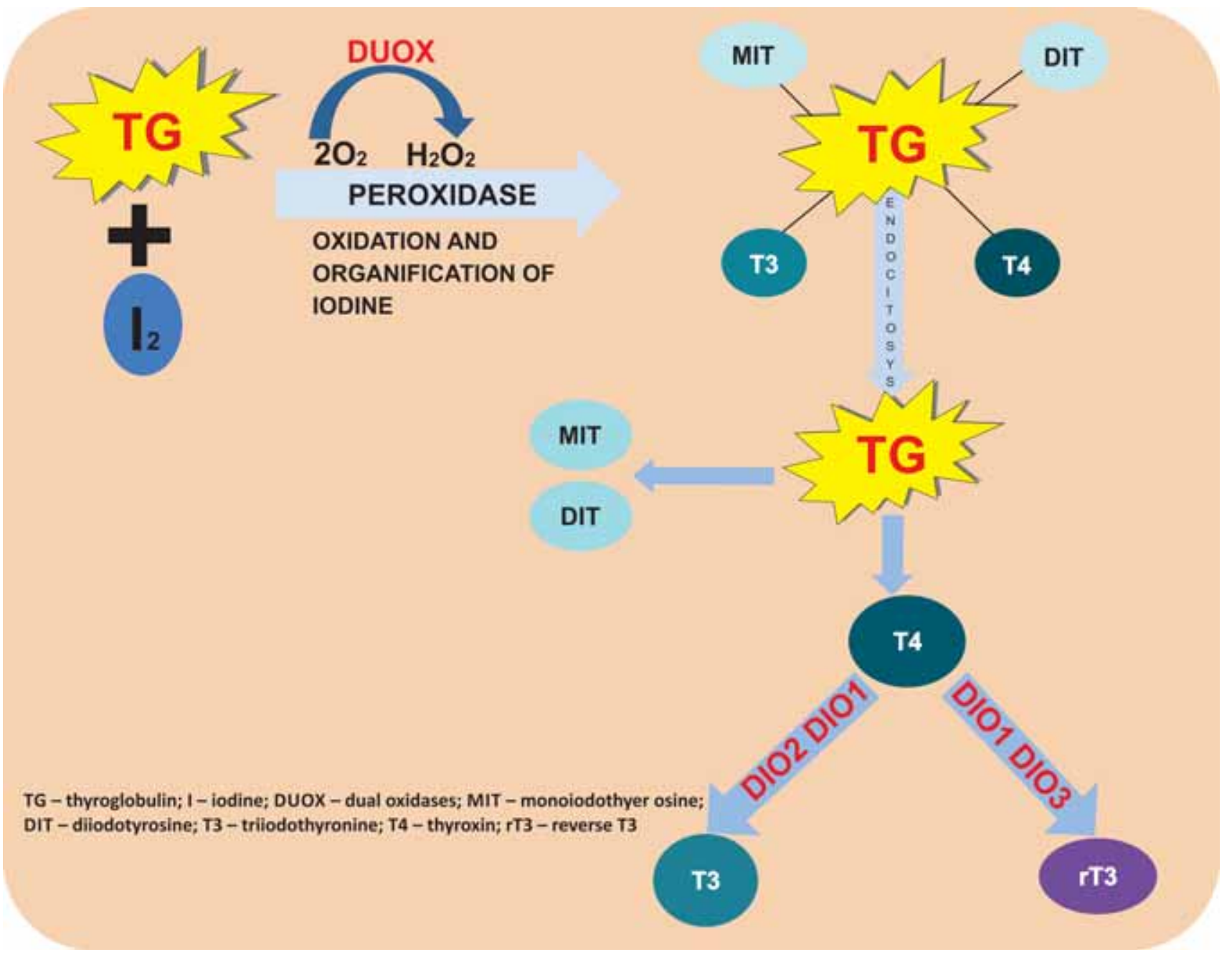

FIGURE 1. Thyroid hormone synthesis

(12). The activity of DIO2 is a source of plasma T3, but also cellular T3 in various tissues $(12,31)$. Studies on mouse model with DIO knocked out showed that serum T3 homeostasis is maintained by compensatory thyroidal T3 secretion (32). These studies also showed the critical and unique role of DIO2 and $\mathrm{DIO} 1$ activity in tissue-specific $\mathrm{T} 3$ production and iodine conservation (32). DIO3 is expressed in most fetal tissues and in the placenta. DIO3 expression drops to undetectable or low levels in most tissues including the heart, shortly after birth. The expression in healthy adult is limited to the skin and brain. Coordinated transient expression of $\mathrm{DIO} 3$ and DIO2 is an aspect of tissue regeneration, in skeletal muscle and liver (33-35). Subsequent expression of DIO2 increases $\mathrm{T} 3$ to drive differentiation while reduction of tissue $\mathrm{T} 3$ levels by DIO3 promotes cell proliferation in regenerating tissue and plays a similar role in tumorigenesis and that is why is classified as an oncofetal protein (36). The homeostasis of cellular T3 can be influenced by differential expression of DIO2 and DIO3 (12).
In cardiac tissue, TH regulate angiogenesis, fibrosis, myocyte shape, energy metabolism, and that is why a reduction of the levels of plasma is one of the hormonal changes associated with advanced heart failure, and other critical illnesses (37-40). Studies on rodent models showed inactivation of TH by DIO3 suggesting that there is an additional mechanism by which reduced $\mathrm{TH}$ action may play a role in heart failure particularly in the early stages of pathological remodeling (41). The proof of this relation is for now lacking (42).

\section{OXIDATIVE STRESS AND THYROID FUNCTION}

The thyroid follicle is the functional structure responsible for $\mathrm{TH}$ biosynthesis, storage and secretion. Follicular thyroid cells are polarized and specialized in the production of T4 and T3 (43). TH are synthesized within the structure of a thyroid-specific protein called $\mathrm{Tg}$, and they remain covalently bound to the primary structure of this molecule un- 
til protein degradation occurs then the hormone is secreted. The first step in TH biosynthesis is the iodination of tyrosyl residues of $\mathrm{Tg}$. Tg is synthesized and secreted into the follicular lumen, and the iodination reaction is believed to occur at the apical membrane of the cells and the colloid interface (44). At the apical surface of the thyrocyte a number of chemical reactions are fundamental for $\mathrm{TH}$ synthesis: iodide oxidation, tyrosyl radical oxidation, $\mathrm{Tg}$ iodination and coupling of iodotyrosines to form the iodothyronines T4 and T3 (43).

The enzymatic reactions that involve the oxidation of substrates depend on the presence of hydrogen peroxide and a peroxidase enzyme that catalyses the process, thyroperoxidase (TPO). In the thyrocyte, the hydrogen peroxide necessary for $\mathrm{TH}$ biosynthesis is generated at the apical surface of the cell through a controlled reaction catalyzed by 2 members of the NADPH oxidases (NOX) family, DUOX1 and DUOX2 $(45,46)$. Presently, the NOX family is composed of seven members: two DUOXes (DUOX1 and DUOX2) and five NOXes (NOX1, NOX2, NOX3, NOX4, and NOX5) (47) (Fig. 1).

Hydrogen peroxide was detected at the apical surface of thyrocytes since 1984 indicating a high level of this oxidizing agent in the thyroid gland. More recently studies confirmed that the thyrocyte is under OS and yet the source of thyroid hydrogen peroxide involved in TH biosynthesis and cell damage has only recently begun to be clarified $(45,48)$.

OS is the unbalance between prooxidant substances production and antioxidant defense systems. The most important prooxidants are the reactive nitrogen species (RNS) and reactive oxygen species (ROS) (49).

In some cell types including thyrocytes, ROS generation could play functional roles (50).

DUOX are essential for TPO catalyzed hormone synthesis. DUOX1 and DUOX2 are present in thyroid and they work in conjunction with maturation factors that allow DUOX enzymes to translocate to the follicular cell membrane and exert their enzymatic activity (DUOXA1 and DUOXA2) (51). A new intracellular ROS generating system recently described in the human thyroid gland is NOX4 (46).

The thyroid hydrogen peroxide generating system began to be elucidated after 2006 , by cloning the maturation factors DUOXA gene (52).

No human DUOX1 mutations have been identified to be related to thyroid dyshormonogenesis, and that is why the exact role of this enzyme in thyroid physiology remains elusive. However, it is believed that DUOX1 could function to overcome the lack of DUOX2 activity because it was observed that some DUOX2 and DUOXA2 mutations lead to transient hypothyroidism (53).

It was demonstrated that hydrogen peroxide generated by DUOX, inhibit TPO activity due to oxidative damage to the enzyme. This could lead to higher cell OS $(53,54)$. If DUOX activity is increased, and the TPO activity is reduced, thyroid tissue could be harmed because hydrogen peroxide is less consumed by the TPO system and produced in higher amounts by DUOX. ROS accumulate, leading to oxidative damage of the thyroid gland $(55,56)$. Interestingly, more recently, it has been showed that the basal activity of DUOX1 and DOUX2 depends on calcium $(57,58)$. Recently it has been demonstrated that the expression and function of DOUX2 in thyroid cells is inhibited by physiological concentrations of $\mathrm{Tg}$ (59).

Another NOX family member, NOX4, was recently described in human thyrocytes (60). The role of NOX4 in thyroid pathophysiology it is not completely elucidated. In contrast with DUOX, NOX4 generates hydrogen peroxide and/or $\mathrm{O}_{2}$ in intracellular compartments such as the endoplasmic reticulum, mitochondria or nucleus $(61,62)$. It was demonstrated that the expression of $\mathrm{NOX} 4$ is upregulated in thyroid cancers, linking NOX4 - dependent hydrogen peroxide generation to cancer development or progression $(63,64)$. In contrast, DUOX2 expression is normal or even decreased in the majority of the differentiated thyroid carcinomas $(65,66)$.

Although many studies have demonstrated that DUOXes are responsible for the hydrogen peroxide synthesis needed in $\mathrm{TH}$ production, other studies (67) suggest a pivotal role for SOD3 as a major hydrogen peroxide-producing enzyme. SODs are hydrogen peroxide producing isoenzymes responsible for balancing the reduction-oxidation reactions in different cellular compartments. According to Laatikainen, Lilja E., et al., SOD3 is highly expressed in normal thyroid, and becomes even more abundant in rat goiter models (67).

Studies suggests that SOD3 is involved in TSH mitogen effect in normal thyrocytes, a pathway disrupted in thyroid cancer and that is why it is believed that SOD3 could be used as a differentiation marker in thyroid cancer (67).

Some hormones, in particular $\mathrm{TH}$, influence antioxidant levels and, on the other hand, OS can modify synthesis, activity and metabolism of hormones. Recent studies showed that OS is related to both systemic inflammation and hormonal derange- 


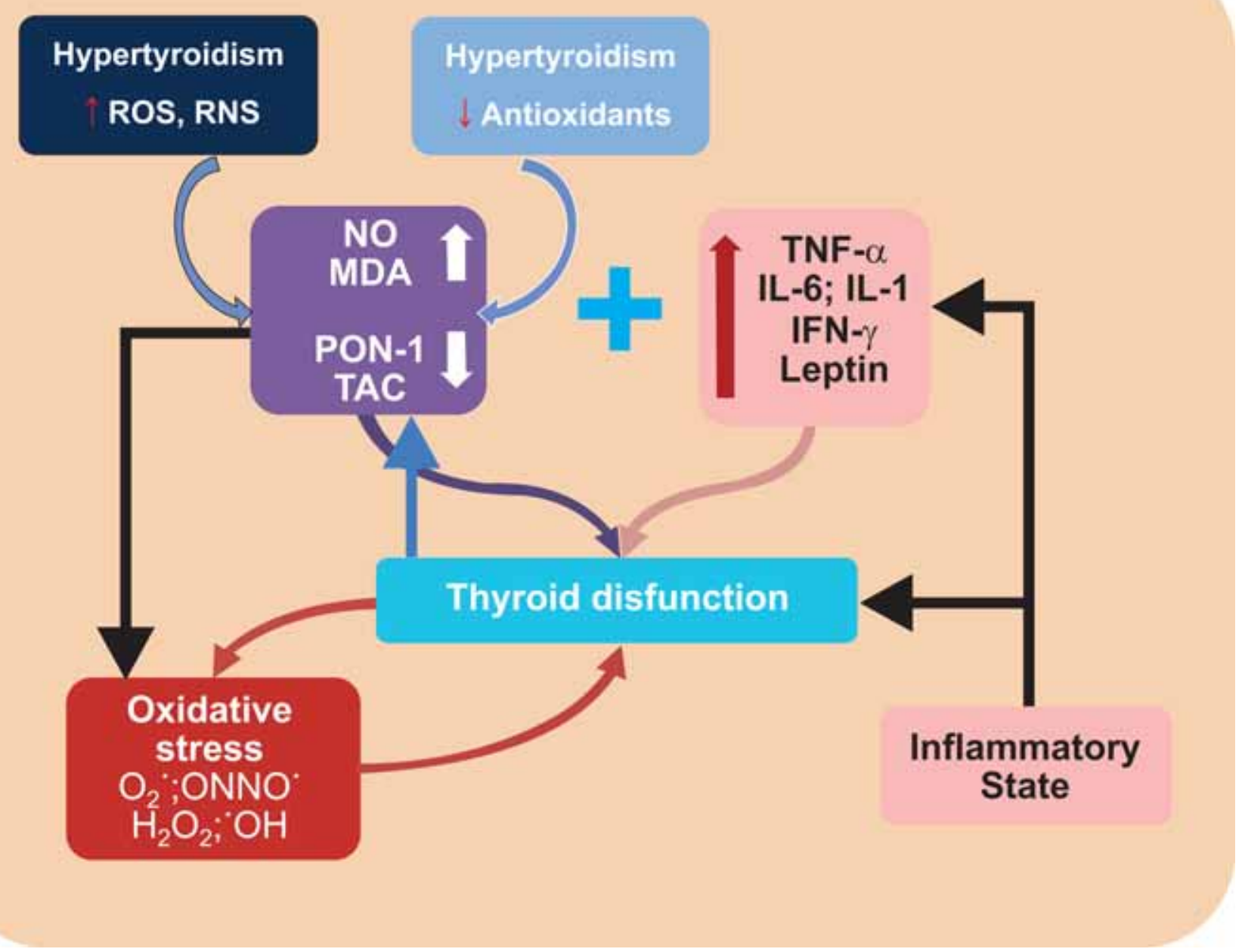

FIGURE 2. Interrelationship between OS, thyroid derangement and inflammation

ROS - reactive oxygen species, RNS - reactive nitrogen species, NO - nitric oxide, MDA - malondialdehyde, PON1 - paraoxonase, TAC - total antioxidant capacity, TNF - tumor necrosis factor, IL - interleukin, IFN - interferon,

$\mathrm{O}_{2}{ }^{-}$- superoxide anion, $\mathrm{ONOO}^{\circ}$ - peroxynitrite, $\mathrm{H}_{2} \mathrm{O}_{2}$ - hydrogen peroxide, ${ }^{\circ} \mathrm{OH}$ - hydroxide (after Mancini A. et al. 2016)

ment. Both hyperthyroidism and hypothyroidism have been shown to be associated with OS and special cases are the autoimmune thyroiditis or the functional picture of low-T3 syndrome, observed in acute and chronic nonthyroidal illness syndrome (NTIS) $(68,69)$.

The role of thyroid in the regulation of the antioxidant systems has been recently reviewed (70). OS has been shown to be associated with both hyperthyroidism and hypothyroidism however, the mechanisms are different: increased ROS production in hyperthyroidism and low availability of antioxidants in hypothyroidism. Some hyperthyroidism complications in target tissues are caused by OS (71). TH per se can act as oxidants and produce DNA damage (72).

The data on hypothyroidism and OS in humans are conflicting. In a group of patients with primary hypothyroidism, Baskol et al. (73) found SOD levels not significantly different from those of con- trols, low activity of paraoxonase (PON-1) and high plasma levels of malondialdehyde (MDA). They found also that the treatment with $\mathrm{TH}$ increased PON-1 activity and decreased MDA levels (73). Dardano, Angela et al. showed that excess TSH directly produces OS (74). Studies on patients with thyroiditis should be, however, interpreted with caution, in that both tissue inflammation and systemic inflammation are present in this autoimmune disorder (29).

The studies available in the literature suggest that the alterations of the pituitary-thyroid axis depend on the severity of the disease, and also on the inflammatory response and the patients nutritional status. They also indicate that low-T3 syndrome is associated with tissue hypothyroidism and OS $(75,76)$ (Fig. 2).

A special role is played by selenium. This essential trace element exerts complex effects on endocrine system, due to its antioxidant capacity. 
Selenium is a cofactor of glutathione peroxidase (GPx) and thioredoxin reductase (TrxR), enzymes that protect the cells from the oxidative damage (77). On the other hand, selenium is involved in the mechanisms of deiodination (78), even though the catalytic mechanisms and the regulation of DIOs by selenium are not fully understood (79). Thus, because of its double function, molecules that compete for this element could connect low-T3 hypothyroidism and OS. OS seems to be an important mechanism underlying the progress of inflammation. TH can have a protective role, modulating antioxidant levels; on the other side, a tissue hypothyroidism can worsen OS (29).

\section{CONCLUSIONS}

In conclusion, there are important biomarkers that could help thyroid affections biochemical diagnosis and patient management in a minimal invasive way. Beside routine thyroid parameters such as TSH, FT4 and FT3, studies showed that Tg level, ratio between TSH and Tg, DIO expression, TPO level are important. Recent studies showed also a correlation between thyroid disorders and OS markers such as NADPH oxidases, DUOX and SOD. The major advantage of measuring those parameters is that they are a minimal invasive way to diagnose thyroid disorders and follow patient evolution.

Conflict of interest: none declared Financial support: none declared

\section{REFERENCES}

1. Hoermann R., Midgley J.E.M., Larisch R. et al. Relational Stability in the expression of normality, variation, and Control of Thyroid Function. Front in endocrinol (Lausanne) 2016; 7:142

2. Petric R., Perhavec A., Gazic B. et al. Preoperative serum thyroglobulin concentration is an independent predictive factor of malignancy in follicular neoplasms of the thyroid gland. J Surg Oncol. 2012; 105.4: 351-356

3. McLeod D.S.A., Cooper D., Ladenson P.W. et al. Prognosis of differentiated thyroid cancer in relation to serum thyrotropin and thyroglobulin antibody status at time of diagnosis. Thyroid, 2014; 24.1 : 35-42.

4. Haymart M.R., Glinberg S.L., Liu J. et al. Higher serum TSH in thyroid cancer patients occurs independent of age and correlates with extrathyroidal extension. Clin endocrinol. 2009; 71.3: 434-439.

5. Sands N.B., Karls S., Rivera J. et al. Preoperative serum thyroglobulin as an adjunct to fine-needle aspiration in predicting well-differentiated thyroid cancer. Otolaryngol Head Neck Surg. 2010; $39.6: 669-673$

6. Pellegriti G., Frasca F., Regalbuto C. et al. Worldwide increasing incidence of thyroid cancer: update on epidemiology and risk factors. J Cancer Epidemiol. 2013; 2013:965212

7. Cho Y.A., Kong S.Y., Lee J. et al. Biomarkers of thyroid function and autoimmunity for predicting high-risk groups of thyroid cancer: a nested case-control study. BMC Cancer. 2014; 14: 873

8. Peterson E., Prithwish D., Robert N. BMI, diet and female reproductive factors as risks for thyroid cancer: a systematic review. PLoS One. 2012; 7.1: e29177

9. Haymart M.R., Repplinger D.J., Leverson G.E. et al. Higher serum thyroid stimulating hormone level in thyroid nodule patients is associated with greater risks of differentiated thyroid cancer and advanced tumor stage. J Clin Endocrinol Metab. 2008; 93.3: 809-814

10. Yeh N.C., Chou C.W., Weng S.F. et al. Hyperthyroidism and thyroid cancer risk: a population-based cohort study. Exp Clin Endocrinol Diabetes 2013;121.07: 402-406.

11. Kim E.S., Lim D.J., Baek K.H. et al. Thyroglobulin antibody is associated with increased cancer risk in thyroid nodules. Thyroid 2010; $20.8: 885-891$

12. Gereben B., Zavacki A.M., Ribich S. et al. Cellular and molecular basis of deiodinase-regulated thyroid hormone signaling. Endocr rev 2008; 29.7: 898-938.

13. Hoermann R., Midgley J.E.M., Larisch R. et al. Homeostatic control of the thyroid-pituitary axis: perspectives for diagnosis and treatment. Front Endocrinol (Lausanne). $2015 ; 6$ : 117 equilibria between free thyroid hormones and pituitary thyrotropin are modulated by various influences including age, body mass index and treatment. Clin Endocrinol. 2014; 81.6: 907-915.

15. Biondi $B$. The normal TSH reference range: what has changed in the last decade? J Clin Endocrinol Metab. 2013; 98(9): 3584-3587.

16. Wartofsky L., Richard A.D. The evidence for a narrower thyrotropin reference range is compelling. $J$ Clin Endocrinol Metab. 2005; 90(9): 5483-5488.

17. Shi L., Li Y., Guan H. et al. Usefulness of serum thyrotropin for risk prediction of differentiated thyroid cancers does not apply to microcarcinomas: results of 1,870 Chinese patients with thyroid nodules. Endocrine 2012; 59.11: 973-980.

18. Azizi G., Keller J.M., Lewis M. et al. Association of Hashimoto's thyroiditis with thyroid cancer. Endocr Relat Cancer. 2014; 21.6: 845-852.

19. Boelaert K., Horaceka J., Holder R.L. et al. Serum thyrotropin concentration as a novel predictor of malignancy in thyroid nodules investigated by fine-needle aspiration. J Clin Endocrinol Metab 2006; 91(11): 4295-4301.

20. Qin J., Yu Z., Guan H. et al. High Thyroglobulin Antibody Levels Increase the Risk of Differentiated Thyroid Carcinoma. Dis markers.

21. Rinaldi S., Plummer M., Biessy C. et al. Thyroid-stimulating hormone, thyroglobulin, and thyroid hormones and risk of differentiated thyroid carcinoma: the EPIC study. J Natl Cancer Inst. 2014; 106.6:097-097

22. Cohen N., Pakdaman M., Rochon L. et al. SP196-Tg: TSH ratio as a tumor marker for thyroid cancer. Otolaryngol Head Neck Surg 2009; 141.3 suppl 1: P165-P165.

23. Wang L., Li H., Yang Z. et al. Preoperative serum thyrotropin to thyroglobulin ratio is effective for thyroid nodule evaluation in euthyroid patients. Otolaryngol Head Neck Surg 2015; 153.1: 15-19.

24. Yazici P., Mihmanli M., Bozkurt E. et al. Which is the best predictor of thyroid cancer: thyrotropin, thyroglobulin or their ratio? Hormones 2016; 15.2: 256-263.

25. Lee E.K., Chung K.W., Min H.S. et al. Preoperative serum thyroglobulin as a useful predictive marker to differentiate follicular thyroid cancer from benign nodules in indeterminate nodules. J Korean Med.Sci. 2012; 27.9: 1014-1018.

26. Cooper D.S., Doherty G.M., Haugen B.R. et al. Management guidelines for patients with thyroid nodules and differentiated thyroid cancer: The American Thyroid Association Guidelines Taskforce. Thyroid 2006; 16.2: 109-142.
14. Hoermann R., Midgley J.E.M., Giacobino A. et al. Homeostatic 2015; 2015: 648670-648670. 
27. Schweizer U., Steegborn C. New insights into the structure and mechanism of iodothyronine deiodinases. J Mol Endocrinol. 2015; 55.3: R37-R52.

28. van der Spek A.H., Fliers E., Boelen A. The classic pathways of thyroid hormone metabolism. Mol Cell Endocrinol. 2017; 458:29-38

29. Mancini A., Segni C.D. Raimondo S. et al. Thyroid Hormones, Oxidative Stress, and Inflammation. Mediators Inflamm. 2016; 2016 : 6757154-6757154

30. Drigo R.A., Bianco A.C. Type 2 deiodinase at the crossroads of thyroid hormone action. Int J Biochem Cell Biol. 2011; 43.10 : 1432-1441.

31. Orozco A., Valverde-R C., Olvera A. et al. lodothyronine deiodinases: a functional and evolutionary perspective. Int $\mathrm{J}$ Endocrinol 2012; 215.2 : 207-219.

32. Galton V.A., Schneider M.J., Clark A.S. et al. Life without thyroxine to 3, 5, 3'-triiodothyronine conversion: studies in mice devoid of the 5'-deiodinases. Endocrinology. 2009; 150.6: 2957-2963.

33. Dentice M., Ambrosio R., Damiano V. et al. Intracellular inactivation of thyroid hormone is a survival mechanism for muscle stem cell proliferation and lineage progression. Cell metab. 2014; 20.6:10381048.

34. Salvatore D., Simonides W.S., Dentice M. et al. Thyroid hormones and skeletal muscle - new insights and potential implications. Nat Rev Endocrinol. 2014; 10.4:206-214.

35. Kester M.H.A., Toussaint M.J.M., Punt C.A. et al. Large induction of type III deiodinase expression after partial hepatectomy in the regenerating mouse and rat liver. Endocrinology 2008; 150.1: 540-545.

36. Dentice M., Antonini D., Salvatore D. Type 3 deiodinase and solid tumors: an intriguing pair. Expert Opin Ther Targets. 2013; 17.11: 1369-1379.

37. de Vries E.M., Fliers E. Boelen A. The molecular basis of the non-thyroidal illness syndrome. J of Endocrino. 2015; 225.3: R67-R81.

38. Gerdes A.M. Restoration of thyroid hormone balance: a game changer in the treatment of heart failure? Amn J Physiol Heart Circ Physiol. 2015; 308.1: H1-H10.

39. Cokkinos D.V., Chryssanthopoulos S. Thyroid hormones and cardiac remodeling. Heart Fail Rev. 2016; 21.4: 365-372.

40. Pingitore A., Nicolini G., Kusmic C. et al. Cardioprotection and thyroid hormones. Heart Fail Rev., 2016; 21(4): 391-399.

41. Pol C.J., Muller A., Simonides W.S. Cardiomyocyte-specific inactivation of thyroid hormone in pathologic ventricular hypertrophy: an adaptative response or part of the problem? Heart Fail Rev. 2010 15.2: 133-142.

42. Janssen R., Muller A., Simonides W.S. Cardiac Thyroid Hormone Metabolism and Heart Failure. EurThyroid J. 2017; 6.3 : 130-137.

43. Bargi-Souza P., Goulart-Silva F., Nunes M.T. Novel aspects of T3 actions on $\mathrm{GH}$ and TSH synthesis and secretion: physiological implications. J Mol Endocrinol. 2017; 59.4: R167-R178.

44. Citterio C.E., Veluswamy B., Morgan S.J. et al. De novo triiodothyronine formation from thyrocytes activated by thyroidstimulating hormone. J Biol Chem. 2017; 292.37: 15434-15444.

45. Virion A., Michot J.L., Deme D. et al. NADPH-dependent H 202 generation and peroxidase activity in thyroid particular fraction. $\mathrm{Mol}$ Cell Endocrinol. 1984; 36.1 : 95-105.

46. Ameziane-El-Hassani R., Schlumberger M., Dupuy C. NADPH oxidases: new actors in thyroid cancer? Nat Rev Endocrinol. 2016; 12.8: 485-494

47. Krause K.H. Tissue distribution and putative physiological function of NOX family NADPH oxidases. Jpn J Infect Dis. 2004; 57.5: S28-9.

48. Carvalho D.P., Dupuy C. Role of the NADPH oxidases DUOX and NOX4 in thyroid oxidative stress. Eur Thyroid J. 2013; 2.3: 160-167.

49. Lancaster J.R.Jr. Nitric oxide in cells. Am Sci. 1992;80: 248-259.

50. Wu C., Zhang Y., Chai L. et al. Oxidative stress, endocrine disruption, and malformation of Bufo gargarizans embryo exposed to sub-lethal cadmium concentrations. Environ Toxicol Pharmacol. 2017; 49: 97-104.

51. Ohye H., Sugawara M. Dual oxidase, hydrogen peroxide and thyroid diseases. Exp Biol Med. 2010; 235.4: 424-433..
52. Grasberger H., Refetoff S. Identification of the maturation factor for dual oxidase evolution of an eukaryotic operon equivalent. J Biol Chem. 2006; 281.27: 18269-18272.

53. Fortunato R.S., Lima de Souza E.C., Ameziane-el Hassani R. et al. Functional consequences of dual oxidase-thyroperoxidase interaction at the plasma membrane. J Clin Endocrinol Metab. 2010; 95.12: 5403-5411

54. Ginabreda M.G., Cardoso L.C., Nobrega F.M. et al. Negative correlation between thyroperoxidase and dual oxidase $\mathrm{H}_{2} \mathrm{O}_{2}-$ generating activities in thyroid nodular lesions. Eur J Endocrinol. 2008; 158.2: 223-227.

55. Song Y., Driessens N., Costa M. et al. Roles of hydrogen peroxide in thyroid physiology and disease. J Clin Endocrinol Metab. 2007; 92.10: 3764-3773.

56. Song Y., Ruf J., Lothaire P. et al. Association of duoxes with thyroid peroxidase and its regulation in thyrocytes. J Clin Endocrinol Metab. 2010; 95.1: 375-382.

57. Ameziane-El-Hassani R., Morand S., BoucherJ.L. et al. Dual oxidase-2 has an intrinsic $\mathrm{Ca} 2+$-dependent $\mathrm{H} 2 \mathrm{O} 2$-generating activity. J Biol Chem. 2005; 280.34: 30046-30054.

58. Rigutto S., Hoste C., Grasberger H. et al. Activation of dual oxidases Duox1 and Duox2 differential regulation mediated by camp-dependent protein kinase and protein kinase C-dependent phosphorylation. J Biol Chem. 2009; 284.11: 6725-6734.

59. Yoshihara A., Hara T., Kawashima A. et al. Regulation of dual oxidase expression and $\mathrm{H} 2 \mathrm{O} 2$ production by thyroglobulin. Thyroid. 2012; 22.10: 1054-1062.

60. Weyemi U., Caillou B., Talbot M. et al. Intracellular expression of reactive oxygen species-generating NADPH oxidase NOX4 in normal and cancer thyroid tissues. Endocr Relat Cancer. 2010; 17.1: 27-37.

61. Hilenski L.L., Clempus R.E., Quinn M.T. et al. Distinct subcellular localizations of Nox1 and Nox4 in vascular smooth muscle cells. Arterioscler Thromb Vascular Biol. 2004; 24.4: 677-683.

62. Ushio-Fukai M. Compartmentalization of redox signaling through NADPH oxidase-derived ROS. Antioxid Redox Signal. 2009; 11.6: 1289-1299.

63. Weyemi U., Lagente-Chevallier O., Boufrateqech M. et al. ROS-generating NADPH oxidase NOX4 is a critical mediator in oncogenic H-Ras-induced DNA damage and subsequent senescence. Oncogene 2012; 31.9 : 1117-1129.

64. Weyemi U., Dupuy $C$. The emerging role of ROS-generating NADPH oxidase NOX4 in DNA-damage responses. Mutat Res. 2012; 751.2: 77-81.

65. Lacroix L., Nocera M., Mian C. et al. Expression of nicotinamide adenine dinucleotide phosphate oxidase flavoprotein DUOX genes and proteins in human papillary and follicular thyroid carcinomas. Thyroid 2001; 11.11: 1017-1023.

66. Weyemi U., Redon C.E., Parekh P.R. et al. NADPH Oxidases NOXs and DUOXs as putative targets for cancer therapy. Anticancer Agents Med Chem. 2013; 13.3: 502-514.

67. Laatikainen L.E., Castellone M.D., Hebrant A. et al. Extracellular superoxide dismutase is a thyroid differentiation marker downregulated in cancer. Endocr Relat Cancer. 2010; 17.3: 785-796.

68. De Groot L.J. Non-thyroidal illness syndrome is a manifestation of hypothalamic-pituitary dysfunction, and in view of current evidence, should be treated with appropriate replacement therapies. Crit Care Clin. 2006; 22.1: 57-86.

69. Mancini A., Raimondo S., Di Segni C. et al. Non-thyroidal illness: physiopathology and clinical implications. Current Topics in Hypothyroidism with Focus on Development InTech, 2013:183-202

70. Mancini A., Giacchi E., Raimondo S. et al. Hypothyroidism, oxidative stress and reproduction. Hypothyroidism-Influences and Treatments. InTech, 2012:117-134

71. Resch U., Helsel G., Tatzber F. et al. Antioxidant status in thyroid dysfunction. Clin Chem Lab Med. 2002; 40.11: 1132-1134.

72. Dobrzyńska M.M., Baumgartner A., Anderson D. Antioxidants modulate thyroid hormone and noradrenaline induced DNA damage in human sperm. Mutagenesis 2004; 19.4: 325-330.

73. Baskol G., Atmaca H., Tanriverdi F. et al. Oxidative stress and enzymatic antioxidant status in patients with hypothyroidism before 
and after treatment. Exp Clin Endocrinol Diabetes. 2007; 115.08: 522-526.

74. Dardano A., Ghiadoni L., Plantinga Y. et al. Recombinant human thyrotropin reduces endothelium-dependent vasodilation in patients monitored for differentiated thyroid carcinoma. J Clin Endocrinol Metab. 2006; 91.10: 4175-4178.

75. Shum A.M.Y., Polly P. Cancer cachexia: molecular targets and pathways for diagnosis and drug intervention. Endocr Metab Immune Disord Drug Targets. 2012; 12.3: 247-259.

76. Suzuki H., Asakawa A., Amitani H. et al. Cancer cachexiapathophysiology and management. J Gastroenterol. 2013; 48.5: 574-594.
77. Balázs C., Rácz K. The role of selenium in endocrine system diseases. Orv Hetil. 2013;1 54.41: 1628-1635.

78. Kuiper G.G.J.M., Kester M.H.A., Peeters R.P. et al. Biochemical mechanisms of thyroid hormone deiodination. Thyroid 2005; 15.8: 787-798.

79. Schweizer U., Towell H., Vit A. et al. Structural aspects of thyroid hormone binding to proteins and competitive interactions with natural and synthetic compounds. Mol Cell Endocrinol. 2017; 458:57-67 